\title{
Adult Combined Hepatocellular Carcinoma and Cholangiocarcinoma
}

National Cancer Institute

\section{Source}

National Cancer Institute. Adult Combined Hepatocellular Carcinoma and

Cholangiocarcinoma. NCI Thesaurus. Code C114987.

A combined hepatocellular carcinoma and cholangiocarcinoma that occurs during adulthood. 